\title{
COVID-19 and Social Distancing: A Pandemic of Advanced Diseases Is at Birth
}

\author{
Hossein Molavi Vardanjani, MPH, PhD ${ }^{1 *}$; Ali Mirzazadeh, MD, PhD²; Golnoush Mahmoudi-Nezhad, MD, MPH ${ }^{3}$ \\ ${ }^{1}$ MD/MPH Program, School of Medicine, Shiraz University of Medical Sciences, Shiraz, Iran \\ ${ }^{2}$ Department of Epidemiology and Biostatistics, University of California San Francisco, San Francisco, CA, USA \\ ${ }^{3}$ Stein Eye Institute, David Geffen School of Medicine, University of California at Los Angeles, Los Angeles, CA, USA
}

$\mathrm{T}$ The pandemic of coronavirus disease 2019 (COVID-19) continues to grow with an unknown end. Currently, healthcare systems are struggling with this pandemic and have postponed most of elective visits and procedures for other diseases. COVID-19 has affected many lives both economically and mentally. ${ }^{1}$

To mitigate the COVID-19 pandemic, social distancing has been implemented in almost all countries worldwide at public places including health care settings. It requires health care systems to triage urgent, semi-urgent, and nonurgent patients. Decreasing contacts between patients and health providers has been enforced by triaging the patients, and by postponing most of in-person visits and elective surgeries. ${ }^{2}$ This strategy has two side effects in long term.

First, using our new recommended triage-based guidelines, we attempt to avoid missing any urgent patient and to postpone non-urgent conditions. However, let's think about "urgent conditions" from the public health point of view and not solely a clinical view. For example, let's consider a poorly educated patient of low socioeconomic status with type 2 diabetes, one of the world's leading causes of preventable death. This patient may be fearful and consequently, avoid referring to a clinic for disease management where there may be exposure to the COVID-19. They may be triaged with telemedicine without a comprehensive examination. Such a paradigm may cause months of delay in follow-up as a non-urgent patient despite needing in-person follow-up. Months later, some patients will present with worsening and advanced disease. Postponing routine follow-up may result in similar scenarios for some of the almost 422 million individuals worldwide with type 2 diabetes.

Second, the development of new diseases will not pause during this long pandemic. Some people may have asymptomatic diseases or diseases with less severe symptoms, and postponing their diagnostic visits and examinations as a result of triage or fear of having an examination will preclude their timely diagnosis.

Both scenarios could lead to a massive burden on patients and health care system such as higher costs of medical and surgical care for advanced disease, a significant increase in need for emergency services, increasing the sequelae of diseases for the patients, and decreasing quality of life for relevant families, higher needs for rehabilitation units and social supports, higher burden on insurance companies, and greater need for subspecialized service.

Moreover, social distancing and public fear or even panic, intensified economic and financial problems for a large number of people (e.g., cutting employees' insurance coverage for cost reduction and laid-off workers), and communication difficulties with people through telemedicine may lead to postponing of appointments by patients or physicians which lead to delayed diagnosis and treatment. This will affect almost all chronic conduction like diabetes. Various sources report that so far, there has been a huge drop in using healthcare service and in prescriptions for patients. ${ }^{3}$

Therefore, another aspect of the current COVID-19 pandemic might be the expected influx of individuals with advanced diseases and the resulting burden on the healthcare systems and patients. We can call this the "pandemic of pandemic-related advanced diseases".

This problem could be further exacerbated in developing countries or underserved populations due to the greater likelihood that optimal guidelines cannot or will not be followed. Without timely and appropriate interventions, many patients lose access to appropriate healthcare.

Strategies are now critical to mitigate the pandemic of pandemic-related advanced diseases. These should include some measures such as the following:

1- Integrated population-based remote screening and case-finding programs for prevalent/detrimental diseases,

2- Follow-up of patients who have canceled their appointments,

3- Population-based remote programs for promotion of health literacy, especially targeting low socially developed subpopulations, 
4- Tailored and highly dynamic public pandemic communication to improve public trust in medical visits,

5- Extending and promoting universal health coverage programs,

6- Separating patients' clinic visits along with strict screening procedures for the COVID-19 (preattendance to clinic or hospital, or at-home-kit tests),

7- Extending technical support for developing countries to develop their appropriate healthcare guidelines during the pandemic,

8- Expanding telemedicine in screening, home monitoring and proper management of patients.

The quantity, quality and also timeliness of healthcare have been highly affected by the COVID-19 pandemic and social distancing. We have to adapt and strengthen the current screening, case-finding, and also patient follow-up programs, and we urgently need to start more programs, especially in deprived populations. As a lesson from the COVID-19 pandemic, in times of public health crises and the conditions in which we implement social distancing, it is necessary to address "emergency" situations from a public health perspective.

\section{Authors' Contribution}

All authors have contributed equally.

\section{Conflict of Interest Disclosures}

None was declared.

\section{Ethical Statement}

None.

\section{References}

1. Munster VJ, Koopmans M, van Doremalen N, van Riel D, de Wit E. A novel coronavirus emerging in China - key questions for impact assessment. N Engl J Med. 2020;382(8):692-694. doi:10.1056/NEJMp2000929.

2. Barsom EZ, Feenstra TM, Bemelman WA, Bonjer JH, Schijven MP. Coping with COVID-19: scaling up virtual care to standard practice. Nat Med. 2020;26(5):632-634. doi:10.1038/s41591020-0845-0.

3. Baum A, Schwartz MD. Admissions to veterans affairs hospitals for emergency conditions during the COVID-19 pandemic. JAMA. 2020;324(1):96-9. doi:10.1001/jama.2020.9972.

Received: June 27, 2020, Accepted: June 30, 2020, ePublished: September 1, 2020

Cite this article as: Molavi Vardanjani H, Mirzazadeh A, Mahmoudi-Nezhad G. COVID-19 and social distancing: a pandemic of advanced diseases is at birth Arch Iran Med. 2020;23(9):651-652. doi: 10.34172/aim.2020.81.

(c) (i) (c) 2020 The Author(s). This is an open-access article distributed under the terms of the Creative Commons Attribution License (http://creativecommons. org/licenses/by/4.0), which permits unrestricted use, distribution, and reproduction in any medium, provided the original work is properly cited. 